\title{
Author Correction: Selective electrocatalysis imparted by metal-insulator transition for durability enhancement of automotive fuel cells
}

Sang-Mun Jung (1), Su-Won Yun, Jun-Hyuk Kim, Sang-Hoon You, Jinheon Park, Seonggyu Lee, Seo Hyoung Chang,

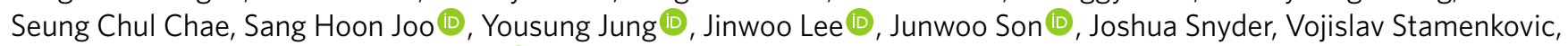
Nenad M. Markovic and Yong-Tae Kim (D)

Correction to: Nature Catalysis https://doi.org/10.1038/s41929-020-0475-4, published online 29 June 2020.

In the version of this Article originally published, the final sentence of the 'Acknowledgements' section was missing. This has now been corrected in the online versions of this Article.

Published online: 3 August 2020

https://doi.org/10.1038/s41929-020-00501-0

(C) The Author(s), under exclusive licence to Springer Nature Limited 2020 